\title{
Evidence of Luttinger-liquid behavior in one-dimensional dipolar quantum gases
}

\author{
R. Citro, ${ }^{1}$ E. Orignac, ${ }^{2}$ S. De Palo, ${ }^{3}$ and M. L. Chiofalo ${ }^{4}$ \\ ${ }^{1}$ Dipartimento di Fisica “E. R. Caianiello” and CNISM, Università degli Studi di Salerno, Salerno, Italy \\ ${ }^{2}$ Laboratoire de Physique de l'École Normale, Supérieure de Lyon, CNRS-UMR5672, Lyon, France \\ ${ }^{3}$ DEMOCRITOS INFM-CNR and Dipartimento di Fisica Teorica, Università Trieste, Trieste, Italy \\ ${ }^{4}$ Classe di Scienze, INFN and CNISM, Scuola Normale Superiore, Pisa, Italy
}

(Received 21 November 2006; published 15 May 2007)

\begin{abstract}
A strongly correlated Luttinger-liquid behavior is found to emerge well beyond the Tonks-Girardeau (TG) regime in a one-dimensional Bose gas with dipolar repulsions at $T=0$, persisting for a wide range of densities. After combining reptation quantum Monte Carlo and bosonization techniques, we provide a unifying theory of the underlying crossover physics, evolving from the TG gas at low density into a classical quasiordered state at high density. The density dependent Luttinger parameters extracted from the numerical data provide all that is needed to determine the low-energy behavior from analytical expressions. Our quantitative predictions, in the whole crossover, for measurable quantities such as the structure factor and the momentum distribution, are estimated to be accessible in underway experiments with ultracold polar molecules.
\end{abstract}

PACS number(s): 03.75.Hh, 05.30.-d, 71.10.Pm, 02.70.Ss

The realization of Bose-Einstein condensation (BEC) in trapped ultracold quantum atomic gases [1] is at the frontier of modern atomic and molecular, optical, and condensedmatter physics [2]. Especially fascinating results arise from the possibility of tuning the atomic interactions. Use of Fano-Feshbach resonances $[3,4]$ to change the magnitude and sign of the $s$-wave scattering length $a$ characterizing the contact interactions, has allowed, e.g., the observation of collapsing Bose condensates [5] and of the crossover from a BEC to a Bardeen-Cooper-Schrieffer-type transition [6].

More recent experiments have demonstrated that the range of the interactions can also be manipulated. Dipole interactions with long-range anisotropic character have been observed in ${ }^{52} \mathrm{Cr}$ atoms [7] after exploiting the large magnetic moments of this atomic species, $\mu_{d} \approx 6 \mu_{B}$ with $\mu_{B}$ being the Bohr magneton. A BEC containing up to $50000{ }^{52} \mathrm{Cr}$ atoms has then been obtained below a transition temperature $T_{c} \simeq 700 \mathrm{nK}[8]$ and its dynamical behavior is being investigated [9]. Promising proposals to tune and shape the dipolar interaction strength in quantum gases of heteronuclear polar molecules have more recently been suggested [10]. Significant theoretical predictions have accompanied such realizations [11]. The stability diagram of anisotropic confined dipolar gases has been predicted to be governed by the trapping geometry $[12,13]$, as corroborated by path-integral quantum Monte Carlo (QMC) studies [14] and discussed in diffusion QMC simulations including the dependence of $a$ on the dipole interaction [15].

Tuning of the interactions can be combined with the enhancement of quantum fluctuations after reducing their dimensionality by, e.g., storing them in elongated traps [16,17], which could be relevant to applications such as precision measurements [18], quantum computing [19], atomtronic quantum devices, and theoretical investigations of novel quantum phase transitions [20].

In the case of quasi-one-dimensional (1D) condensates with short-range interactions, a rich phenomenology is known to emerge despite the absence of broken symmetries [21]. Bosons are known to arrange in a Luttinger-liquid state, with single particles being replaced by collective density excitations $[22,23]$. Strong repulsion may also lead to the fer- mionization of interacting bosons in the so-called TonksGirardeau (TG) regime [24-26]. Experiments in elongated traps have provided evidence for such 1D fluctuations [16].

For fermionic 1D models, the case of interactions falling off as $1 / x^{\alpha}$ has been studied by many authors [27,28], concluding that for $\alpha>1$ the long-range part of the interaction decays rapidly enough to preserve the linear energy dispersion $\omega(k)=u|k|$ typical of short-range models. Extrapolation of this result to bosons would suggest that 1D Bose gases with short enough ranged $1 / x^{\alpha}$ interactions $(\alpha>1)$ can behave at low energy as Luttinger liquids [22]. Unlike Fermi systems, however, proving such behavior is a nontrivial task since a nonperturbative approach is required.

An interesting question thus arises for quasi-1D condensates with dipolar interactions, that is, whether the combination of enhanced quantum fluctuations and strong repulsion are sufficient to drive the BEC in a super strong-coupling regime, beyond the TG state [29]. More recent diffusion QMC simulations [30] for a homogeneous 1D dipolar Bose gas have revealed a crossover behavior with increasing linear density, from a liquidlike superfluid state to an ordered, normal state with particles localized at lattice sites. For low values of the density, the system is seen to behave as a Tonks-Girardeau gas [24]. A quantitative and unifying theory revealing the nature of the Bose dipolar liquid in the whole crossover region is, however, missing, which is needed to predict the behavior of observable quantities in the experiment.

In this Rapid Communication we provide such a theory, giving evidence for a robust Luttinger-liquid behavior of the 1D dipolar Bose gas in the crossover region well beyond the Tonks-Girardeau state, gradually evolving from a TG gas at low density into a classical quasiordered state at high density, consistent with the absence of a quantum phase transition to a solid [31]. By comparing theoretical results from a bosonization approach against reptation quantum Monte Carlo simulations [32] we are able to provide a unifying theory of the underlying crossover physics. After analyzing the scaling behavior of the static structure factor expected for a Luttinger liquid, we find a small exponent $K<1$ at inter- 
mediate to high-density values, signaling very strong repulsions at short distances. The knowledge of Luttinger parameters is then exploited to determine observable properties of the fluid in a parameter range accessible to experiments. To the best of our knowledge this is the first demonstration of such a strongly correlated liquid behavior in Bose gases with dipolar interactions within a continuum model $[33,34]$.

We consider $N$ atoms or molecules of mass $M$ and permanent dipole moments arranged along a line. For the purposes of the present work, we assume $a \rightarrow 0$ after, e.g., exploiting a Fano-Feshbach resonance. In effective Rydberg units $\mathrm{Ry}^{*}$ $=\hbar^{2} /\left(2 M r_{0}^{2}\right)$ the Hamiltonian is

$$
H=\left(-\frac{1}{r_{s}^{2}} \sum \frac{\partial^{2}}{\partial x^{2}}+\frac{1}{r_{s}^{3}} \sum \frac{1}{\left|x_{j}-x_{j}\right|^{3}}\right)
$$

where the effective Bohr radius $r_{0} \equiv M C_{d d} /\left(2 \pi \hbar^{2}\right)$ is defined in terms of the interaction strength $C_{d d}$. One has $C_{d d}=\mu_{0} \mu_{d}^{2}$ and $C_{d d}=d^{2} / \epsilon_{0}$ for magnetic $\mu_{d}$ and electric $d$ dipole moments, respectively, with $\mu_{0}$ and $\epsilon_{0}$ the vacuum permittivities. The governing dimensionless parameter $r_{s}=1 /\left(n r_{0}\right)$ contains the linear particle density $n$ and the unit length $r_{s} r_{0}$.

We analyze the low-energy structural properties of the fluid. They can be accessed in experiments with atomic gases by means of, e.g., Bragg scattering techniques. Here, we infer them from QMC numerical simulations of the static structure factor $S(k)$ on a box with size $L$ and periodic boundary conditions. In terms of the density operator $\hat{n}$ this is $S(k)=\int_{0}^{L} d x e^{-i k x}\langle\hat{n}(x) \hat{n}(0)\rangle$.

An accurate size-scaling analysis of $S(k)$ is needed for our purposes. To this aim we resort to the reptation quantum Monte Carlo (RQMC) of Baroni and Moroni [32], which in essence is a path-integral technique at zero temperature giving direct access to the ground-state wave function. This makes the extraction of the ground-state properties conceptually simple and that of the correlation functions in imaginary time immediate [35], with the additional possibility of determining the excitation spectrum. We use a trial wave function made up of a two-body Jastrow factor $\psi_{\text {trial }}(R)$ $=\Pi_{i<j} \exp \left[u\left(\left|x_{i}-x_{j}\right|\right)\right]$. Removal of the short-range divergences in $H \psi_{\text {trial }} / \psi_{\text {trial }}$ due to the potential energy, is obtained after imposing the cusp condition, which yields $u(x \rightarrow 0)$ $=-\sqrt{r_{s} / x}[36]$.

The RQMC data for $S(k)$ are reported in Fig. 1 for $N$ $=40$ and different values of $n r_{0}=0.01,50,100$, and 1000 . Free fermionlike behavior, typical of the Tonks gas, is seen at low-density values $\left(n r_{0}=0.01\right.$ in Fig. 1). Quasi-Bragg peaks emerge at reciprocal lattice vectors $k / n=2 \pi m$ ( $m$ integer). Their number increases with increasing $n r_{0}$, evidencing the occurrence of a quasiordered state. Investigation of the RQMC data at different values of $N$ shows that the peak heights scale as $N^{\alpha_{m}}$ with $\alpha_{m}<1$ being an exponent depending on $m$, while their intensity diverges with the system size.

Both these characteristics are reminiscent of Luttingerliquid behavior and therefore we turn on analyzing the data within a bosonization description. In terms of the conjugate bosonic operators $\phi$ and $\Pi$, the Hamiltonian for a Luttinger liquid reads [21]

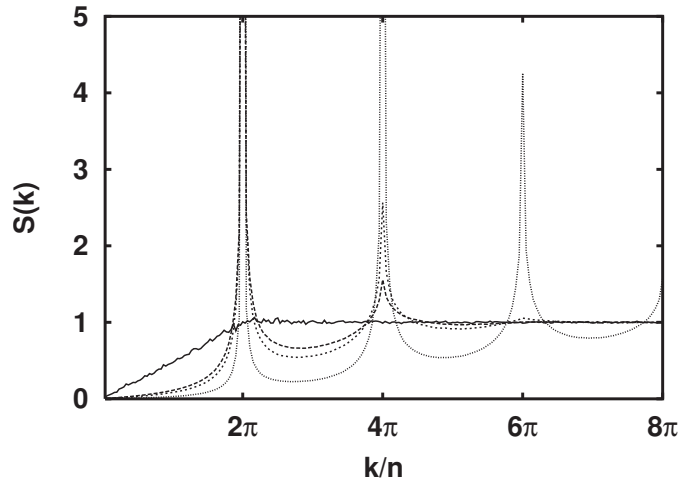

FIG. 1. $S(k)$ in dimensionless units for a dipolar gas with $N$ $=40$ particles and different values of $n r_{0}=0.01,50,100$, and 1000 . Decreasing slopes as $k \rightarrow 0$ and the emergence of additional peaks correspond to increasing $n r_{0}$ values.

$$
H=\hbar \int \frac{d x}{2 \pi}\left[u K(\pi \Pi)^{2}+\frac{u}{K}\left(\partial_{x} \phi\right)^{2}\right],
$$

where $K$ is the Luttinger exponent and $u$ is the sound velocity. Knowledge of $K$ and $u$ allows analytical expressions for physical quantities of interest. The structure factor $S(k)$ can be analytically calculated inserting in the definition of $S(k)$ above the bosonized expression of the density operator $\hat{n}(x)=-\frac{1}{\pi} \partial_{x} \phi+\sum_{m \neq 0} A_{m} n e^{2 i[m \phi(x)-\pi n x]}[21,22]$, where $A_{m}$ are nonuniversal dimensionless constants. In dimensionless units for $k, S(k)$ turns out to be $S_{0}(k)=K k /(2 \pi)$ for $k \rightarrow 0$. For $k$ close to $k=2 \pi m$ on the scale of the cutoff parameter $\pi / \alpha$, we find [37]

$$
S(k) \approx \sum_{m \neq 0} A_{m}^{2} n^{2} \Sigma_{m}(k \pm 2 \pi m),
$$

where $\Sigma_{m}(k)$ is explicitly given as

$$
\begin{aligned}
\Sigma_{m}(k)= & L\left(1-e^{-2 \pi \alpha / L}\right)^{2 m^{2} K} \sigma\left(k ; m^{2} K, L\right) \\
& \times{ }_{2} F_{1}\left(m^{2} K, m^{2} K+|k| L /(2 \pi) ; 1+|k| L /(2 \pi) ; e^{-4 \pi \alpha / L}\right),
\end{aligned}
$$

where $\sigma\left(k ; m^{2} K, L\right) \equiv \Gamma\left[m^{2} K+|k| L /(2 \pi)\right]\left[\Gamma\left(m^{2} K\right) \Gamma(1\right.$ $+|k| L /(2 \pi))]^{-1} ;{ }_{2} F_{1}$ is the Gauss hypergeometric function.

The low- $k$ linear behavior of $S(k)$ can be fitted to extract the Luttinger $K$ at different densities. The resulting $K(n)$ is displayed in Fig. 2. The inset shows $S_{0}(k)$ for selected densities, where decreasing slopes correspond to increasing values of $n r_{0}=50,100,1000$, the symbols representing the RQMC data and the lines the Luttinger prediction $S_{0}(k)$ $=K k /(2 \pi) . K$ remains below the Tonks-Girardeau limit in a wide range of densities, signaling the occurrence of strong correlations. As $n r_{0}<1, K$ tends to the TG limit $K=1$. In the opposite high-density limit, $K$ tends to vanish following the classical-theory prediction $K \sim \pi\left[6 \zeta(3) n r_{0}\right]^{-1 / 2}$. In essence, strong repulsions at short distance produce a TG-type fermionization while the remaining longer-range part pushes the fermionized gas into a strongly correlated phase.

For $\alpha \ll L, \Sigma_{m}(k)$ gets a simplified form depending on whether $\gamma \equiv m^{2} K<1 / 2$ or $\gamma>1 / 2$ as $L \rightarrow \infty$. For $\gamma<1 / 2$, 


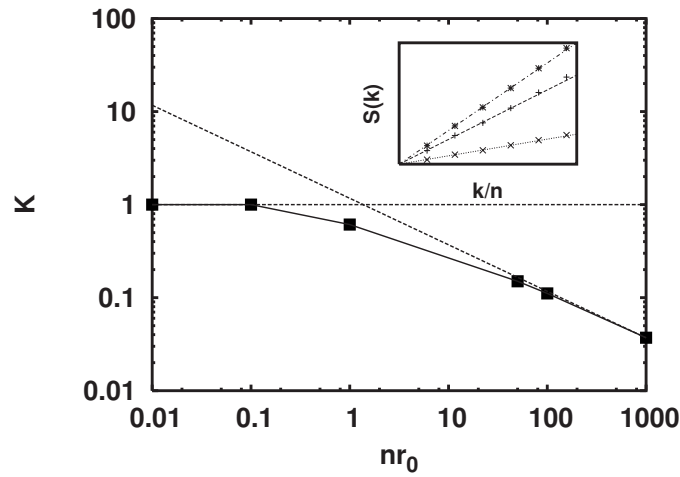

FIG. 2. Luttinger exponent $K$ obtained from fitting the RQMC data for $S(k \rightarrow 0) \equiv S_{0}(k)$. For comparison, the curves $K(n \rightarrow 0)=1$ and $K(n \rightarrow \infty)=\pi\left[6 \zeta(3) n r_{0}\right]^{-1 / 2}$ are also displayed as dashed lines, corresponding to the Tonks-Girardeau and to the classical predictions. The solid line is a guide to the eye. Inset: $S_{0}(k)$ at selected $n r_{0}$ (see text).

$\Sigma_{m}(k) \sim L^{1-2 m^{2} K} \sin \left(\pi m^{2} K\right) \beta(K ; L) \quad$ with $\quad \beta(K ; L) \equiv \Gamma(1$ $\left.-2 m^{2} K\right) \Gamma\left[m^{2} K+k L /(2 \pi)\right] / \Gamma\left[1-m^{2} K+k L /(2 \pi)\right]$, indeed revealing the presence of quasi-Bragg peaks at $|k|=2 \pi m$ and a scaling behavior in the vicinity thereof. Peak number increases with decreasing $K$, and peak intensities diverge as $L^{1-2 m^{2} K}$. For $\gamma>1 / 2$ instead, $\Sigma_{m}(k \rightarrow 0) \sim 2^{1-2 \gamma} \Gamma(2 \gamma$ -1) $\Gamma(\gamma)^{-2}$ and the peaks disappear.

Besides the linear $k$ behavior, evidence of a Luttinger liquid comes also from the scaling behavior of $S(k)$. Figure 3 displays the comparison between the RQMC $S(k)$ close to $k=2 \pi$ towards the quasiordered region with $n r_{0}=50$, and the predictions of the Luttinger-liquid theory Eqs. (3) and (4) with $m=1$. We actually plot $S(k) / S(2 \pi n)$ for different $N$ values and verify that they indeed collapse on a single curve. This signature of Luttinger-liquid behavior against the RQMC data is robust in the whole crossover region. This is the central result of the present work. Similar results are indeed found at the remaining density values above $n r_{0}=1$, while the scaling is absent below $n r_{0}=1$. The same scaling analysis holds for the second peak at $k=4 \pi$ when it is present. At $k=6 \pi(m=3)$, we have $2 m^{2} K>1$ for $K<0.055$ and the divergent peak is seen to show up in the simulation only for the data set with $n r_{0}=1000$, as expected.

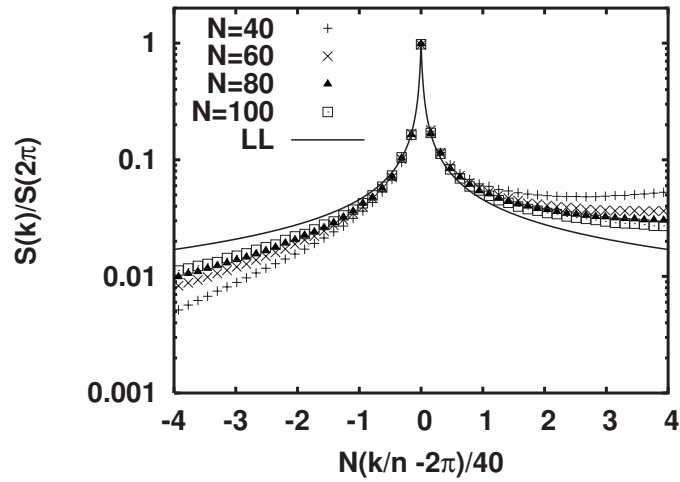

FIG. 3. $S(k) / S(2 \pi)$ in $n^{-1}$ units vs $N(k-2 \pi) / 40$ at $n r_{0}=50$ for different $N$ values (see legend). Symbols represent RQMC data; the solid line is the Luttinger-liquid prediction.
The peak asymmetry visible in Fig. 3 results from band curvature effects. Asymmetric $S(k)$ are also obtained in models possessing a nonlinear spectrum such as CalogeroSutherland models [34] and can be viewed as anharmonic phonon effects [38].

As a consistency check, we have verified that the $K$ values obtained from the RQMC $S(k)$ agree with those determined from the RQMC energy per particle $\epsilon$, as follows. Since Hamiltonian (1) is invariant under Galilean boosts [22], we have $u K=\hbar \pi n / M$. Within the bosonization procedure $[21,22]$, the compressibility is $\chi=K\left(\hbar \pi u n^{2}\right)^{-1}$. Comparing this result with the definition of $\chi, \chi^{-1}=n^{2}(n \epsilon(n))^{\prime \prime}$, we obtain $K=\hbar \pi \sqrt{n /\left[M(n \epsilon(n))^{\prime \prime}\right]}$. This result stems from the trivial fact that the exact RQMC satisfies the compressibility sum rule. However, we have formally demonstrated that our Luttinger-liquid scheme satisfies the $\chi$ sum rule too.

The knowledge of $K(n)$ allows us to determine the momentum distribution $n(k)$, which in current BEC experiments can be accessed after analyzing, e.g., time-of-flight images. Since in the continuum the boson creation operator is represented by $\psi_{B}^{\dagger}(x) \sim \exp \left[-i \pi \int^{x} d y \Pi(y)\right]$ [21], we find that $n(k) \propto k^{1 / 2 K-1}$ as $L \rightarrow \infty$. As the density increases, the divergence of $n(k)$ at $k=0$ is expected to be gradually reduced until it disappears for $K<1 / 2$.

In conclusion, we have shown that dipolar gases in a reduced 1D geometry are promising candidates to observe unusually strong correlations well beyond the Tonks-Girardeau regime, evolving from a TG gas at low density to a quasiordered state at high density. In the whole crossover region, the system remains in a Luttinger-liquid phase, consistent with the absence of a quantum phase transition to a solid [31]. Clear testing of the latter conclusion can be provided by the excitation spectrum [39] where, from preliminary data, our combination of bosonization and RQMC techniques in a continuum can be successfully applied. Observation of the Luttinger liquid in the crossover region is within the reach of current and future experiments in elongated traps. Here, the effective 1D character of trapped dipolar gases is governed by the condition $n^{-1} \gg l_{\perp}$, with $l_{\perp} \equiv\left[r_{0} /\left(4 a_{\perp}\right)\right]^{1 / 5} a_{\perp}$ ensuring the suppression of forward scattering [10]. Values of the transverse oscillator length $a_{\perp}=\sqrt{\hbar /\left(M \omega_{\perp}\right)}$ related to the harmonic angular frequency $\omega_{\perp}$ can be pushed to $50 \mathrm{~nm}$. Then, dipolar $\mathrm{SrO}$ molecular gases in elongated traps are promising candidates, as we might have $l_{\perp} \approx 0.2 \mu \mathrm{m}$ while $r_{0} \approx 240 \mu \mathrm{m}$, yielding access to $n r_{0}$ values up to the quasiordered regime. For dipolar ${ }^{52} \mathrm{Cr}$ gases instead we can have $l_{\perp} \approx 31 \mathrm{~nm}$ while $r_{0} \approx 4.8 \mathrm{~nm}$, yielding access to $n r_{0}$ values remaining down in the Tonks-Girardeau regime. For ultracold $\mathrm{SrO}$ dipolar quantum gases created in such quasi-1D conditions, we quantitatively predict the density-dependent low-energy properties of the liquid as determined by the Luttinger parameters and provide guiding analytical expressions. A power-law decay of the peak heights in $S(k)$ and a flattening of $n(k)$ as $n r_{0} \gg 1$ are expected fingerprints of the Luttinger liquid.

We thank Paolo Pedri for very useful suggestions on the quasi-1D realizations. We also thank G. C. La Rocca 
for useful discussions. S.D.P. is indebted to Dr. S. Moroni for helpful discussions and QMC support. M.L.C. has benefited from discussions with L. Pitaevskii, S.
Stringari, and S. Giorgini. R.C., S.D.P., and E.O. acknowledge hospitality from SNS during the conduct of this work.
[1] M. A. Anderson, J. R. Ensher, M. R. Matthews, C. E. Wieman, and E. A. Cornell, Science 269, 198 (1995); K. B. Davis et al., Phys. Rev. Lett. 75, 3969 (1995); C. C. Bradley, C. A. Sackett, J. J. Tollett, and R. G. Hulet, ibid. 75, 1687 (1995).

[2] Bose-Einstein Condensation in Atomic Gases, in Proceedings of the International School of Physics "Enrico Fermi" Course CXL, edited by M. Inguscio, S. Stringari, and C. Wieman (IOS Press, Amsterdam, 1999).

[3] H. Feshbach, Ann. Phys. 5, 357 (1958); U. Fano, Phys. Rev. 124, 1866 (1961).

[4] S. Inouye, M. R. Andrews, J. Stenger, H.-J. Miesner, D. M. Stamper-Kurn, and W. Ketterle, Nature (London) 392, 151 (1998).

[5] J. Roberts et al., Phys. Rev. Lett. 86, 4211 (2001); T. Weber, J. Herbig, M. Mark, H.-C. Nägerl, and R. Grimm, Science 299, 232 (2003).

[6] C. A. Regal, M. Greiner, and D. S. Jin, Phys. Rev. Lett. 92, 040403 (2004); M. Bartenstein, A. Altmeyer, S. Riedl, S. Jochim, C. Chin, J. H. Denschlag, and R. Grimm, ibid. 92, 120401 (2004); M. W. Zwierlein et al., ibid. 92, 120403 (2004); T. Bourdel, L. Khaykovich, J. Cubizolles, J. Zhang, F. Chevy, M. Teichmann, L. Tarruell, S. J. J. M. F. Kokkelmans, and C. Salomon, ibid. 93, 050401 (2004); M. W. Zwierlein, J. R. Abo-Shaeer, A. Schirotzek, C. H. Schunck, and W. Ketterle, Nature (London) 435, 1047 (2005).

[7] J. Stuhler, A. Griesmaier, T. Koch, M. Fattori, T. Pfau, S. Giovanazzi, P. Pedri, and L. Santos, Phys. Rev. Lett. 95, 150406 (2005).

[8] A. Griesmaier, J. Werner, S. Hensler, J. Stuhler, and T. Pfau, Phys. Rev. Lett. 94, 160401 (2005).

[9] S. Giovanazzi, P. Pedri, L. Santos, A. Griesmaier, M. Fattori, T. Koch, J. Stuhler, and T. Pfau, Phys. Rev. A 74, 013621 (2006).

[10] H. P. Büchler et al., Phys. Rev. Lett. 98, 060404 (2007).

[11] See, e.g., M. Baranov, L. Dobrek, K. Góral, L. Santos, and M. Lewenstein, Phys. Scr., T T102, 74 (2002).

[12] L. Santos, G. V. Shlyapnikov, P. Zoller, and M. Lewenstein, Phys. Rev. Lett. 85, 1791 (2000); K. Goral, K. Rzazewski, and T. Pfau, Phys. Rev. A 61, 051601(R) (2000).

[13] S. Giovanazzi, A. Görlitz, and T. Pfau, Phys. Rev. Lett. 89, 130401 (2002) suggest to use time-dependent external fields for stability.

[14] K. Nho and D. P. Landau, Phys. Rev. A 72, 023615 (2005).

[15] D. C. E. Bortolotti, S. Ronen, J. L. Bohn, and D. Blume, Phys. Rev. Lett. 97, 100402 (2006).

[16] D. Hellweg, S. Dettmer, P. Ryytty, J. Arlt, W. Ertmer, K. Sengstock, L. Santos, G. V. Shlyapnikov, and M. Lewenstein, Acta Phys. Pol. B 73, 781 (2001); A. Görlitz et al., Phys. Rev. Lett. 87, 130402 (2001); S. Richard, F. Gerbier, J. H. Thywissen, M. Hugbart, P. Bouyer, and A. Aspect, ibid. 91, 010405 (2003).

[17] For a collection: Quantum Gases in Low Dimensions, edited by L. Pricoupenko, H. Perrin, and M. Olshanii, special issue of J. Phys. IV 116 (2004).

[18] See, e.g., M. A. Kasevich, Science 298, 1363 (2002); I. Carusotto, L. Pitaevskii, S. Stringari, G. Modugno, and M. Inguscio, Phys. Rev. Lett. 95, 093202 (2005).

[19] See, e.g., I. Bloch, Nat. Phys. 1, 23 (2005), and references therein.

[20] K. Goral, L. Santos, and M. Lewenstein, Phys. Rev. Lett. 88, 170406 (2002); H. Pu, W. Zhang, and P. Meystre, Phys. Rev. Lett. 87, 140405 (2001); H. Pu et al., Phys. Rev. Lett. 89, 090401 (2002).

[21] See, e.g., T. Giamarchi, Quantum Physics in One Dimension (Oxford University Press, Oxford, UK, 2004).

[22] F. D. M. Haldane, Phys. Rev. Lett. 47, 1840 (1981).

[23] H. Moritz, T. Stoferle, M. Kohl, and T. Esslinger, Phys. Rev. Lett. 91, 250402 (2003); M. A. Cazalilla, J. Phys. B 37, S1 (2003); D. Petrov et al., J. Phys. IV 116, 3 (2004).

[24] M. Girardeau, J. Math. Phys. 1, 516 (1960).

[25] T. D. Schultz, J. Math. Phys. 4, 666 (1963).

[26] On the possible realization of the TG regime see, e.g., B. Paredes et al., Nature (London) 429, 277 (2004); T. Kinoshita, T. Wenger, and D. S. Weiss, Science 305, 5687 (2004).

[27] Y. Tsukamoto and N. Kawakami, J. Phys. Soc. Jpn. 69, 149 (1999).

[28] H. Inoue and K. Nomura, J. Phys. A 39, 2161 (2006).

[29] G. E. Astrakharchik, J. Boronat, J. Casulleras, and S. Giorgini, Phys. Rev. Lett. 95, 190407 (2005).

[30] A. S. Arkhipov, G. E. Astrakharchik, A. V. Belikov, and Yu. E. Lozovik, JETP Lett. 82, 39 (2005).

[31] To rule out the quantum phase transition one should extend the Mermin and Wagner theorem, Phys. Rev. Lett. 17, 1133 (1966), to 1D fluids at $T=0$; see L. Pitaevskii and S. Stringari, J. Low Temp. Phys. 85, 377 (1991).

[32] S. Baroni and S. Moroni, Phys. Rev. Lett. 82, 4745 (1999).

[33] Similar results are known for hard-core lattice models [G. Gómez-Santos, Phys. Rev. Lett. 70, 3780 (1993); 73, 1053(E) (1994)], while for continuum models only in the case with $1 / r^{2}$ repulsions [F. Calogero, J. Math. Phys. 10, 2191 (1969); 10, 2197 (1969); B. Sutherland, ibid. 12, 246 (1971); 12, 251 (1971)].

[34] E. R. Mucciolo, B. S. Shastry, B. D. Simons, and B. L. Altshuler, Phys. Rev. B 49, 15197 (1994).

[35] S. De Palo, S. Conti, and S. Moroni, Phys. Rev. B 69, 035109 (2004).

[36] We obtain the same results also using either the Luttinger form $u(x) \propto \sin ^{\alpha}(\pi x / L)$ or Gaussians centered on the lattice sites as trial wave functions.

[37] For further details: E. Orignac et al. (unpublished).

[38] F. D. M. Haldane, J. Phys. C 14, 2585 (1981).

[39] S. Giovanazzi and D. H. J. O’Dell, Eur. Phys. J. D 31, 439 (2004) predict a roton spectrum within a quasi-1D mean-field calculation. 